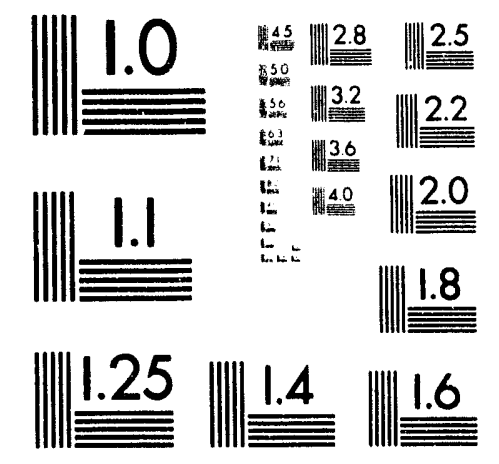



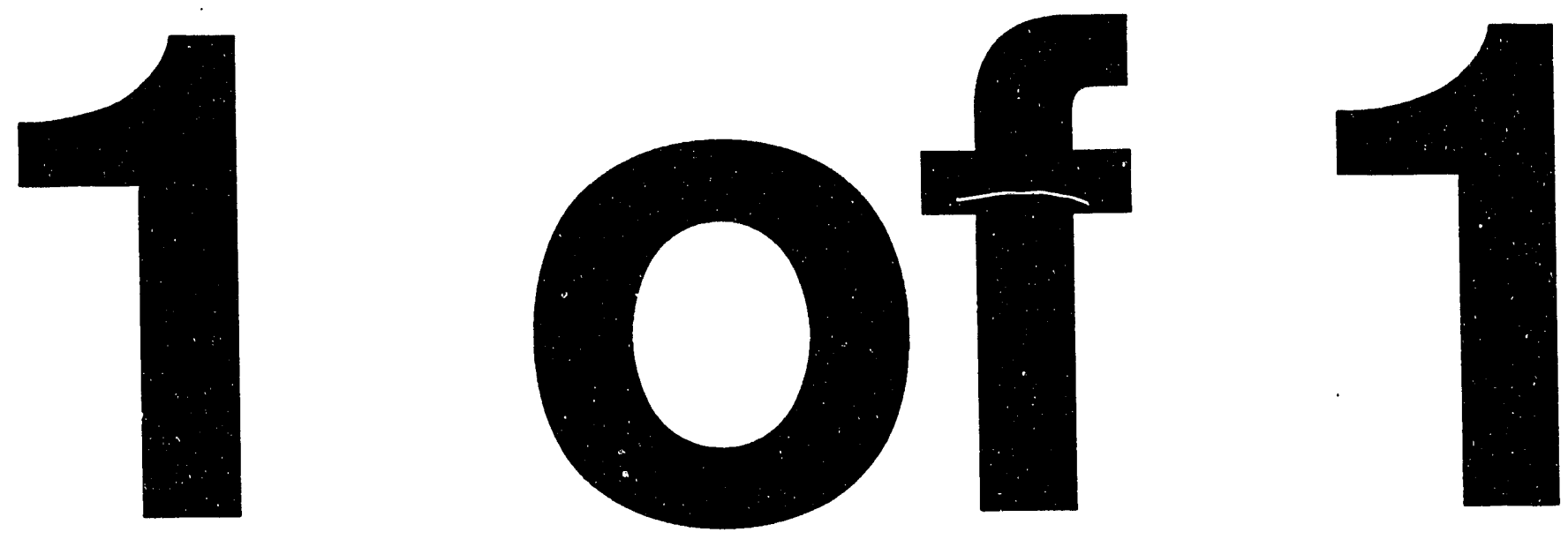
UCRL-JC-113319

PREPRINT

\title{
Vectorized Presentation-Level Services for Scientific Distributed Applications
}

\author{
L.C. Stanberry \\ M.L. Branstetter \\ D.M. Nessett
}

This paper was prepared for submittal to

Supercomputer '93

Portland, OR

November 15-19, 1993

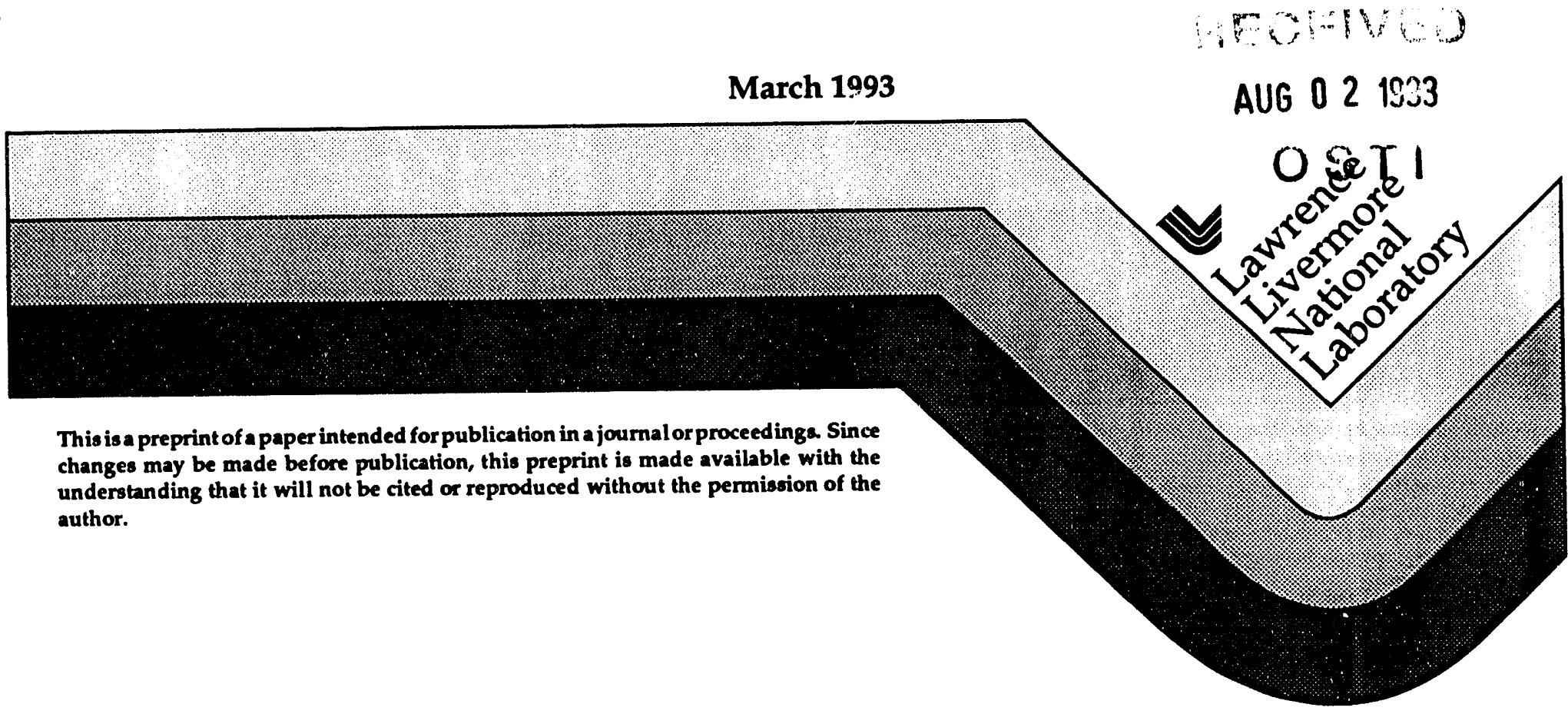




\section{DISCLAIMER}

This document was prepared as an acciunt of work sponsored by an agency of the United States Government. Neither the United Stales Government nor the University of California nor any of their employees, makes any warranty, express or implied, or assumes any legal liability or responsibility for the accuracy, completeness, or usefulness of any information, apparatus, product, or process disclosed, or represents that its use would not infringe privately owned rights. Reference herein to any specific commercial products, process, or service by trade name, trademark, manufacturer, or otherwise, does not necessarily constitute or imply its endorsement, recommendation, or favoring by the United States Govemment or the University of California. The views and opinions of authors expressed herein do not necessarily state or reflect those of the United States Government or the University of California, and shall not be used for advertising or product endorsement purposes. 


\title{
Vectorized Presentation-Level Services for Scientific Distributed Applications
}

\author{
L.C. Stanberry, M.L. Branstetter and D.M. Nessett \\ Lawrence Livermore National Laboratory, \\ Livermore, California 94550
}

\begin{abstract}
The use of heterogeneous distributed systerns is a promising approach to significantly increase computational performance of scientific applications. However, one key to this strategy is to minimize the percentage of time spent by an application moving data between machines. This percentage is composed of two parts: 1) the time to translate data between the formats used on different machines, and 2) the time to move data over the network that interconnects the machines. Previous work suggests that data format conversion activity, generally known as presentation-level services, is by far the more costly of the two.
\end{abstract}

In this paper we describe how vectorization can be used to improve presentation-level performance in scientific applications by one or two orders of magnitude over the conventional approach. While others have recognized the advantages of vectorized data format conversion, we describe how to automate this process so that an application programmer need not explicitly call vectorization routines. We explore the impact of presentation-level vectorization on software portability, programming efficiency and protocol standards. We compare our performance results with those of two other popular distributed application programming tools and then summarize the lessons we have learned during the course of our research.

\section{INTRODUCTION}

There are several different ways to employ parallelism in scientific computations. The use of massively parallel machines has received considerable attention and has generated some notable successes. Many of these successes, however, have come about in the solution of so-called embarrassingly parallel problems, such as Monte Carlo simulations and ray tracing graphics.

Another way to apply parallelism to scientific computational problems is to divide a calculation into several algorithms, where each runs optimally on a diffurent computer architecture $[3,6]$. For example, consider a problem that has both a hydrodynamics calculation and a Monte Carlo calculation. Experience shows that for certain classes of problems, the hydrodynamics calculation runs best on a shared memory multiprocessor, while the Monte Carlo calculation runs best on a massively parallel distributed memory machine [9]. The obvious strategy to achieve the best computational performance is to partition and then distribute the computation, running the hydrodynamics on a shared memory system and the Monte Carlo on a large distributed memory system.

In order for this approach to work effectively, the ratio of computation time taken by each partition to the time taken to move data between partitions must be high. Recent advances in networking technology make it possible to move large volumes of data between machines in a relatively short period of time. Networks capable of achieving close to 1 gigabit per second are currently undergoing field test. While process-to-process communications throughput will be somewhat less than 1 gigabit per second due to operating system and protocol overhead, sufficient residual bandwidth will remain to support the kinds of distributed calculations described above.

However, another problem remains. In many cases the data formats of machines are significantly different. For example, a CRAY X-MP, Y-MP or Y-MP C90, which are classic examples of a shared memory multiprocessor, support a floating point format that is significantly different than that supported on most existing massively parallel distributed memory machines. Furthermore, a CRAY supports integers that are 64 bits in length, while most massively parallel distributed memory machines support only 32 bit integers. This implies that data moved between machines may need to be converted from the sending machine's format into the receiving machine's format.

Communication system architects now know that the time to perform data conversion dominates the time to move data between machines of different architectures, given the availability of high-performance communication networks [2]. In terms of a standard communications architecture, data conversion occurs at the presentation-level. Thus, the feasibility of distributing scientific applications across heterogeneous machines rests, to a certain extent, on the cfficiency of presentation-level services. Improving the efficiency of presentation-level services expands the applicability of distributed computing to a wider range of problems.

With this in mind, we decided to explore ways to make presentation-level activity as small a proportion of computing in a distributed application as possible. Our main focus is on scientific distributed applications; thus, we concentrate on techniques to improve the efficiency of 
converting scientific data, i.c., integers and reals. We describe our investigations concerning the vectorization of presentation-level services for scientific data. Our results include not only the performance improvements we were able to obtain by vectorizing the conversion of integers and reals, but also the implications such an approach has on software portability, programmer productivity, and protocol standardization issues.

In the next section we discuss issues of vectorized data format conversion that affect distributed application development and use. Section 3 describes our approach to each of these issues. We compare the performance of our approach to that of two other software development environments in section 4 . In section 5 we summarize the lessons we have learned from our research and suggest arcas of future investigation.

\section{VECTORIZED PRESENTATION-LEVEL SERVICES : ISSUES}

The job of the presentation-level is to relieve the distributed application programmer from concerns about differences in data representation on machines supporting the application. Two presentation-level issues significantly interact with vectorization: 1) the presentation-level programming interface, and 2) the presentation-level protocol architecture.

\subsection{The presentation-level programming interface}

There are three basic types of programming interface for presentation-level services: 1) a program library, 2) a stub compiler, and 3) an embedded language. Each approach has variations that allow the vectorization of presentation-level services. However, the most popular interfaces in the first two categories do not currently support vectorization and are architected in a way that makes its addition problematic.

\subsubsection{Program libraries}

A program library interface provides access to procedures that encode or decode data into a standard format suitable for transmission between machines. Some libraries also allow the programmer to build up more complicated data structures, such as arrays, from base data types by calling special constructor routines [7].

The most popular presentation-level interface based on a program library, Sun Microsystem's XDR library [7], was not written with vectorization in mind. To send an array of integers or reals using the XDR library, the programmer makes a call to a routine called $x d r_{-}$vector(). One of the arguments to $x d r$ vector() is a pointer to another routine, for example $x d r$ int $(\overline{)}$ or $x d r$ float (), that encodes/decodes the element type of the array. Since for each array element the procedure that encodes/decodes the array calls a procedure that encodes/decodes the array elements, a vectorizing $\mathrm{C}$ compiler does not convert the $x d r$ vector() call into vectorized code.

While it is possible to explicitly call a vectorized routine to convert data into an array of bytes and then transmit them using the XDR library, this moves the responsibility for presentation-level services to the application programmer, decreasing productivity. The programmer for each distributed application must become familiar with the concepts of the presentation-level, as well as use specialized routines that convert data to/from an appropriate intermediate representation.

Moreover, if a program is intended to run on several machines, some of which do not have vectorization hardware, this approach encourages non-portable programs. To accommodate vectorization, either the programmer must \#ifdef the vectorized and non-vectorized versions of the data conversion code, or write two versions of the conversion procedures. In addition, if machines of different architecture provide vector hardware with different interfaces, further \#ifdef sections or procedures are required, one for each architecture on which the application runs. This increases the complexity of application programs, decreasing application programmer productivity.

Another popular system that uses a programming library to access presentation-level services is PVM ${ }^{1}$ [4]. The PVM interface accepts data in the form of arrays, so vectorized format conversion would be possible, although it is not presently implemented. PVM rovides XDR encode/decode functionality by calling standard XDR encode/decode procedures. Consequently, vectorization of PVM presentation-level services would require a reimplementation of XDR within the PVM code set.

In addition to the problems they introduce with respect to vectorization, programming library interfaces to presentation-level services force the application programmer to design miniature presentation-level protocols when sending complex data, such as elaborate records or arrays of records. These protocols are represented by nested calls to library constructor routines and base type translation routines that result in complicated and hard to maintain application source code. Furthermore, interoperability belween applications sending and receiving the same complex data is problematic, since implementors may not choose to represent this data by the same set of nested library calls, resulting in incompatible protocols.

\subsubsection{Stub compilers}

A stub compiler $[5,8]$ produces a procedure, called a stub, that translates data structures represented in a machine's

1 This paper concentrates on PVM 2.4.1, not PVM 3.0, which was released just prior to the submission deadline. However, we belicve our results are valid for either version. 
internal representation $10 /$ rom a standard intermediate representation during the course of sending or receiving a remote procedure call (RPC). The programmer writes a description of a stub in terms of the procedure and its arguments, places it along with other stub descriptions in a module called an interface, and executes the stub compiler, providing the interface module as input. For each stub description, the compiler generates two stubs - one for sending the procedure call and the other for receiving it.

Since stubs are generated by a common stub compiler, they relieve the application programmer from designing and implementing miniature presentation-level protocols to communicate complex data structures. Stubs generated from the same interface represent data with the same protocol mappings, which guarantecs interoperability even if application code is written by different implementors.

While it would be possible for stub compilers to output vectorizable code, to our knowledge such compilers do not exist. Perhaps one reason is the difficulty in automatically generating vectorizable code that can be processed by a large number of vectorizing compilers. For example, a programmer annotates a program to inform a typical vectorizing $C$ compiler that there are no dependencies between pointers referenced inside a loop. However, at present there are no standard annotations used by these compilers ${ }^{2}$. Consequently, a stub compiler would have to generate a sequence of \#ifdef code sections, one section for each $\mathrm{C}$ compiler that the stub generator supports, in order for its code to be portable between different machines. Such an approach would greatly increase stub compiler complexity, leading to significantly higher maintenance costs.

Another possible approach is to place vectorized conversion routines in the RPC runtime library. This eliminates any increased complexity in the stub compiler, placing the burden of managing the vectorized source code with the runtime librarian. Selection of the appropriate source code could take place when the RPC runtime is generated, eliminating the overhead of producing vectorized code each time an application stub is compiled.

As with program library interfaces, the stub compiler approach has characteristics that decrease application programmer efficiency. In particular, stubs are designed, written, compiled and maintained in a separate code development stream from that used to design, write, compile and maintain application programs. From this perspective they are very much like traditional program libraries, except that it is highly unlikely that the stubs from one application are usable in others. Hence, the application programmer lakes on the role of a program librarian in addition to other

2 Currently, the Numerical C Extensions Group of ANSI $\mathrm{X} 3 \mathrm{~J} 11$ is developing a standard annotation for vectorizing C compilers. responsibilities. This increases the complexity of the code development process.

In addition, the application programmer is forced to use an unfamiliar style of programming when implementing distributed application servers. A server program must register its remote procedures with a runtime system dispatcher, rather than accept messages from correspondents and implement the associated semantics directly. Morcover, many stub compilers choose the identifiers of stubs and their parameter types [10], which is acsthetically irritating and introduces application program maintenance problems. For example, some stub compilers use the name of the interface to construct the name of the stubs it generates. Changing the interface name forces the application programmer to modify all of the distributed application modules that call stubs of that interface. This increases program maintenance costs.

\subsubsection{Embedded language}

An embedded language approach to presentation-level scrvices uses special declarations and statements embedded in a program to express the operations and data structures communicated between application processes [1]. These statements complement those of the host programming language and allow programmers to express data translation and transmission activitics in a way that smoothly integrates them into the normal flow of an otherwise conventional program. Data structures that are arbitrarily complex can be clearly expressed with an embedded language, an advantage it enjoys over a program library interface.

Programs containing embedded language statements are first translated by a preprocessor, which converts the embedded language statements into code expressed in the host programming language. The output of the preprocessor is then compiled by a conventional compiler and linked with a runtime library. This strategy allows the programmer to express his presentation-level service requests in a natural way within a program and yet does not require the development of a new compiler, which normally is a costly and lengthy endeavor.

Onc approach to vectorizing the presentation-level services offered by an cmbedded language places vectorized conversion routines for arrays of base types in the runtime library. Instead of generating code loops to convert each element of an array, the preprocessor generates a call to an appropriate array conversion routine that is vectorized or not, depending on the machine on which it is compiled. This strategy simplifies the preprocessor, which need not be written with vectorization in mind at all. The vectorized routines in the runtime system still must contain \#ifdef code segments or be separate routines with the appropriate annotations for the routines to be portable across different vectorizing compilers. In this regard the embedded language approach resembles the use of vectorized routines in the RPC runtime library of a stub compiler. 


\subsection{The presentation-level protocol architecture}

Certain characteristics of the presentation-level protocol dramatically affect the efficiency of vectorized presentationlevel services. In fact, unless the presentation-level message formats satisfy certain constraints, vectorization may not even be possible.

A vector operation assumes its argument vectors are arrays of regularly located memory locations. While vector units can operate on arguments that are scattered in memory, they allow only a very limited form of scattering. Each element of the vector must be a fixed number of memory locations away from its nearest neighbor. Furthermore, this fixed number, the vector's stride, must be constant for all its elements.

The data structures supported by a program library, stub compiler or embedded language can be chosen to satisfy the stride condition of vector operations. Consequently, vectorized conversion is possible no matter how complicated the data structure, as long as it is represented in memory properly, a condition satisfied by most presentation-level interfaces.

However, conversion translates the data structure from its machirs representation to/from its intermediate representation as expressed in a presentation-level protocol. In urder to vectorize the conversion, the protocoi representation of the data structure must also be regularly laid out in memory.

There are three major presentation-level protocols currently in use : 1) XDR, 2) NDR and 3) ASN. ${ }^{3}$. Both the XDR and NDR message formats represent arrays as contiguous data items. Consequently, vectorized routines that convert to/from these message formats are straightforward to writc.

ASN.1, on the other hand, uses a type-length-value style of encoding in which the type, length and value field lengths depend on the value being represented. Arrays of ASN.1 elements do not in general map to a representation regularly laid out in memory.

However, ASN.1 provides for the definition of private data representations, which are standardized only within the domain of a particular application. This allows the definition of ASN.1 data types that represent arrays as contiguous data elements and allows these types to be used as arguments to vectorized operations.

\footnotetext{
${ }^{3}$ Actually, ASN.1 is an Abstract Syntax Notation of the ISO presentation-level protocol, rather than the protocol itself. However, for the purposes of this discussion the distinction is unimportant.
}

\section{VECTORIZED PRESENTATION-LEVEL SERVICES IN ELROS}

ELROS is an cmbedded language with an associated preprocessor and runtime system [1]. We chose an cmbedded language approach because of its advantages with respect to programmer efficiency. We rejected the use of a programming library because our clients required the communication of complex data structures and we wished to relic ve them from the work of designing miniature presentation-level protocols. We rejected a stub compiler approach because we felt our clients would object to dealing with more than one code development stream as well as finding dispatcher-based programming disagrecable.

ELROS preprocesses C code embedded with ELROS type declarations and statements, and generates $C$ code which contains calls to the ELROS runtime routines to perform the indicated communication. ELROS type declarations are type descriptions or mappings to ASN.1 types. The ELROS preprocessor generates a $C$ typedef for each declared type. Ordinary $\mathrm{C}$ statements can be used to declare and assign objects of the declared ELROS types. ELROS statements describe communication (sends and receives) of objects of these types. The preprocessor generates $C$ code to transmit the ASN.1 encoding for the object values, where the cncoding of primitive data type values is performed by ELROS runtime routines. For example, in order to transmit an array of values, the ELROS preprocessor normally generates a loop which calls a runtime routine to encode and transmit one element of the array at a time.

ELROS uses BER encoding of ASN.1 by default. In this encoding, an array is a sequence of encoded values, where each element of an array is encoded as a tag-length-value triple. These triples are not uniform in size since the number of bytes needed to encode the length-value part of the encoding varies with the actual value to encode. Hence, the encoding process for BER is not amenable to vectorization.

ELROS permits the user to specify an enhanced BER encoding for a given communication, hereafter designated as LER, for Livermore Encoding Rules. The basic concept of LER is to use a private encoding that specifies a uniform encoding sizc for array elements in order to allow optrinization of the encoding process. Instead of using an ASN.1 sequence or set tag for the array, LER encoding uses an ASN.1 private type as the tag. Instead of using a separate lag and length for each element, there is only one tag and length for the entire array. That is, the protocol used is taglengih-value where the value field is structured as an elemsize-vector pair. The lag is the private type that designates the type of the array elements (integers or reals). The length is the total number of bytes for elemsize-vector. The elemsize is encoded in a single byte, and indicates the byte size of individual array elements. The number of 
elements can then be deduced from (length-1)/elemsize. The vector is the sequence of encoded values of the array elements, each of which occupy elemsize bytes.

The ELROS preprocessor recognizes and treats integer and real arrays differently from other arrays. Instead of generating a loop as described carlier, it generates a call to a runtime routine to transmit the entire array. If normal $B E R$ encoding was specified for communication, this routine will do the standard BER encoding, one element at a time. If LER encoding was specified, it will attempt to vectorize or optimize the translation of the entire array of values.

LER needs to choose a representation of the array element values which, like the BER encoding, is the intermediate form transmitted. The intermediate form used is big endian, and 2's complement for integers or IEEE for reals. The ELROS runtime routine that performs the encoding makes use of the ELROS type information to determine what size integer or real to use as encoding: $1,2,4$, or 8 byte integers, and 4 or 8 byte IEEE.

There are obvious advantages to using this intermediate form if it happens to be identical to the representation used on the host machine. The LER encoding algorithm is vectorizable on vector machines like the CRAY, and also allows optimizations such as block copies on non-vector machines. Further, the LER algorithm attempts to minimize any copying between buffers. The performance improvements from using LER encoding are discussed below.

Although LER encoding is very promising for improved performance, there are some limitations on its use that need to be noted. Since LER uses a private type encoding, it is not interoperable with other services providing ASN.I encodings such as ISODE, unless the ISODE application provides the decoding from the private types used for LER encoded arrays. It was one of the design goals of ELROS that it would be compatible with other ASN.1 protocol services. The ASN.1 protocol would need to be extended to standardize the "vector" encoding used by LER in order to support this intcroperability.

Also, since the intermediate form used is for common-sized integers and reals, ELROS types that specify extended ("non-native") sizes are always encoded using BER encoding. This limitation is not considered scrious as applications using non-native sizes will be the exception rather than the rule. Our immediate concern was to address the needs of the most common scientific applications, which we anticipate will use native sizes.

\section{PERFORMANCE}

We evaluated the performance of ELROS on a sample application that transmitted an array of $30(0,0)(0)$ clements (4 byte integers or 8 byte reals) between a client and server. The elient sends the array to the server, and the server sends the same array back to the client. We measured the CPU time taken to complete the send and receive by the client, since this includes the encoding and decoding time needed. In measuring the CPU time, we intended to capture only the time spent in doing the data conversions, but as we indicate below, some network communication time also was measured.

The host machines that we used for our evaluation were a Sun4 SPARC station and a CRAY X-MP. We measured performance between two Suns (Sun-Sun), between two CRAYS (CRAY-CRAY), and between the Sun and the CRAY (Sun-CRAY). We also evaluated the difference between performance on the Suns and CRAYs when the client and scrver were on the sarne machine, or on different machines, giving an indication of the potential effect of onmachine versus off-machine network traffic.

We compared the ELROS performance for BER and LER encoding, as a base measure of the improvement of LER over BER encoding. We then compared the performance of ELROS LER encoding to XDR and PVM. While PVM uses the standard XDR routines, it also introduces overhead, as the measurements below show. The comparisons with PVM were done two ways: the default PVM use, where the user allows PVM to do the encoding using XDR, and a more sophisticated use where the user invoked library routines to do the encoding in a vectorized manner, and then used "raw" mode to send the encoded array.

It is worth noting that ELROS vectorized routines perform more than just the translation of the data, however, as they also provide error checking for ELROS types. For example, if the ELROS type specifies that a real type is to use 10 bits for the exponent, the ELROS send routine will check that all values sent are representable with a 10 bit exponent, even though IEEE64 format will allow 11 bits and CRAY real format will allow 15 bits.

The results of these comparisons are tabulated below. We present primarily the Sun-CRAY test results as we feel they are more representative of expected applications within a helerogeneous computing environment. We give a sampling of the other results to indicate the equal or surpassed performance achieved when both client and server are on the same host. For the Sun-CRAY tests, we give the times on both the Sun and CRAY. In all cases, we present the times for integer arrays and for real arrays. The times quoted are averaged from results of multiple executions of the same test. All times are measured in seconds.

Table 1 shows that, as expected, there was considerable improvement in our own performance using LER cncoding. With LER encoding, we achieved a specdup of 10 on the Suns and 10() on the CRAYs. 


\begin{tabular}{|l|c|c|c|c|}
\cline { 2 - 5 } \multicolumn{1}{c|}{} & \multicolumn{2}{c|}{ BER encoding } & \multicolumn{2}{c|}{ LER encoding } \\
\cline { 2 - 5 } \multicolumn{1}{c|}{} & Sun & CRAY & Sun & CRAY \\
\hline Integers & 20.5 & 9.2 & 1.76 & 0.09 \\
\hline Reals & 47.2 & 19.7 & 3.87 & 0.18 \\
\hline
\end{tabular}

Table 1 - Sun-CRAY time in seconds for ELROS BER vs. L.ER encoding

Table 2 shows there was additional performance improvement noted when communicating between two processes on the same host. Since the times shown also indicate some improvement in on-machine vs. off-machine times, there is some contribution in our Sun-CRAY times for off-machine network traffic. We also suspect that different buffer sizes on the two machines may cause an increase in the Sun-CRAY times.

\begin{tabular}{|l|c|c|c|c|}
\cline { 2 - 5 } \multicolumn{1}{c|}{} & \multicolumn{2}{c|}{ BER encoding } & \multicolumn{2}{c|}{ LER encoding } \\
\cline { 2 - 5 } \multicolumn{1}{c|}{} & Sun-Sun & CRAY-CRAY & Sun-Sun & CRAY-CRAY \\
\hline Integers & 20.0 & 9.2 & 1.14 & 0.05 \\
\hline Reals & 45.5 & 19.1 & 2.28 & 0.07 \\
\hline
\end{tabular}

Tát!e 2 - Sun-Sun and CRAY-CRAY time in seconds for ELROS BER vs. LER encoding

Table 3 shows the results of ELROS LER cncoding compared to XDR and PVM. ELROS performs as well or better than XDR on the Sun, and is at least an order of magnitude faster on the CRAY than XDR. The results are even better when compared with PVM, which is not surprising since PVM calls XDR to do its encoding. The time to convert reals on Suns using either XDR or PVM is less than that for integers. This unc xpected result occurs because of a system dependent optimization, which reduces the number of procedure calls per real array element, but has no effect on the number of procedure calls per integer array clement.

\begin{tabular}{|l|c|c|c|c|c|c|}
\cline { 2 - 7 } \multicolumn{1}{c|}{} & \multicolumn{2}{c|}{ ELROS } & \multicolumn{2}{c|}{ XDR } & \multicolumn{2}{c|}{ PVM } \\
\cline { 2 - 7 } \multicolumn{1}{c|}{} & Sun & CRAY & Sun & CRAY & Sun & CRAY \\
\hline Integers & 1.76 & 0.09 & 8.77 & 3.50 & 12.67 & 3.52 \\
\hline Reals & 3.87 & 0.18 & 5.09 & 3.84 & 8.14 & 3.84 \\
\hline
\end{tabular}

Table 3 - Sun-CRAY time in seconds for ELROS, XI)R, and PVM

PVM doesn't attempt to vectorize the encoding, so we tried doing the encoding before calling PVM, using vectorized routines supplied on the CRAY, and using the raw send/receive modes with PVM. Table 4 shows that EL.ROS outperforms even vectorized PVM, although the times on the CRAY are nearly identical.

\begin{tabular}{|l|c|c|c|c|}
\cline { 2 - 5 } \multicolumn{1}{c|}{} & \multicolumn{2}{c|}{ ELROS } & \multicolumn{2}{c|}{ Vechorized PVM } \\
\cline { 2 - 5 } \multicolumn{1}{c|}{} & Sun & CRAY & Sun & CRAY \\
\hline Integers & 1.76 & 0.09 & 12.67 & 0.12 \\
\hline Reals & 3.87 & 0.18 & 8.14 & 0.19 \\
\hline
\end{tabular}

Table 4 - Sun-CRAY time in seconds for ELROS and vectorized PVM

\section{SUMMARY AND FUTURE RESEARCH}

Our goal in this investigation was to determine the feasibility of automating the vectorization of the data format conversion used in communicating between machines. We have argued that it is undesirable for the burden of this vectorization to be placed on the user, and that was our motivation for making this automatic for the user.

Our results have demonstrated that this approach is feasible, and delivers the performance improvements anticipated. We believe our results also support our choice of using an cmbedded language approach as superior to other existing presentation-level services for programmer efficiency and case of use, with respect to code development and maintenance, for reasons stated previously.

However, our current implementation of vectorized presentation-level services does imply some restrictions on the interoperability of ELROS with other ASN.1 protocol services. Our current automation of vectorization is not yet generally applicable to all data types. Both of these limitations are what we hope to address in future work.

The private ASN.1 type encoding that we have utilized necds to become standard if it is to be interoperable with other ASN.1 protocol services. More experience with "vector" encodings will be necded to facilitate a demand for the appropriatc extensions to this standard.

LER encoding in ELROS is currently limited to arrays of integers and reals. We are investigating extensions to the LER encoding that will also optimize encoding of arrays of any primitive type. We are also investigating extensions for the encoding of arrays of ELROS constructed types (Records and Unions). The LER conversion routines for integer and real arrays already support a non-unit stride between elements that will be needed to exiend conversions to constructed data types. Additional extensions to the ASN.1 protocol will be used, with more private type encodings, 10 accommodate these extensions.

\section{A(KNOWLED(BMENTS}

A number of people have worked on the ELROS project, helping with bolh its design and implementation. Jimmy (iuse was one of the principle architects of ELROS and the developer of a prototype implementation, much of which 
was incorporated into the production version. Gary Long and Ashley Allen contributed to some of the carly ELROS design work. Robyne Sumpter worked on parts of the runtime system and Rob Shaw converted the preprocessor parser to an ANSI C grammar. Mark Boolootian is working on optimizing communications performance using techniques different from, but complimentary to, the work described here. We expect his efforts to produce significant results. We also thank Mark for running some preliminary tests that demonstrated the significant performance gains possible by vectorizing the ELROS encode/decode logic.

"Work performed under the auspices of the U.S. Department of Energy by the Lawrence Livermore National Laboratory under contract number W-7405-ENG-48."

\section{REFERENCES}

[1] M.L. Branstetter, J.A. Guse and D.M. Nessett, "ELROS - An Embedded Language for Remote Operations Service", Proceedings of the 1992 IFIP International Conference on Upper Layer Protocols, Architectures and Applications, Vancouver, B.C., May 27 - 29, 1992, pp. 31-45.

[2]David D. Clark and David L. Tennenhouse, "Architectural considerations for a new generation of protocols," SIGCOMM '90 Symposium : Communication Architectures and Protocols, Sept. 24-27, 1990, Association for Computing Machinery, pp. 2(x)-2()8.

[3] Paul F. Dubois and Dan M. Nessctl, "Distributed Scientific Computing in the DOE: Issues and Approaches", UCRL-JC-106533, Lawrence Livermore National Laboratory, April 9, 1991.

[4] G.A. Geist and V. S. Sunderam, "Network-Based Concurrent Computing on the PVM System," Concurrency: Practice and Experience, Vol. 4, No. 4, Junc, 1992, pp. 293311.

[5]B. J. Nelson, "Remote Procedure Call," Tech. Rep. CSL81-9, Xerox Palo Alto Rescarch Center, Palo Alto, Calif., 1981.

16] Dan Nessett and Rich Zwakenberg, "Distributing Scientific Computations Over Multiple Machines," in Buffer, the Newsletter of the National Energy Research Supercomputer Center, December, 1990.

17] "Network Programming," Part No. 8()()-1779-()5, Dec. 3, 1987, Sun Microsystems Inc., Mountain Vicw, CA.

[8] "OSF DCE 1.0 Application Development Guide; Revision 1," Dec. 27, 1991, Open Software Foundation, Cambridge, MA.
[9] James A. Rathkopf, William R. Martin, Amitava Majumdar and Maria Litvin, "Experiences with Different Parallel Programming Paradigms for Monte Carlo Particle Transport Leads to Portable Toolkit for Parallel Monte Carlo," to appear in Procecdings of The Joint International Conference on Mathematical Methods and Supercomputing in Nuclear Applications, Kongresszentrum, Karlsruhe, Germany, April 19-23, 1993.

[10]Marshall T. Rose, Julian P. Onions and Colin J. Robbins, "The ISO Development Environment : User Manual (version 7)," July 19, 1991. 

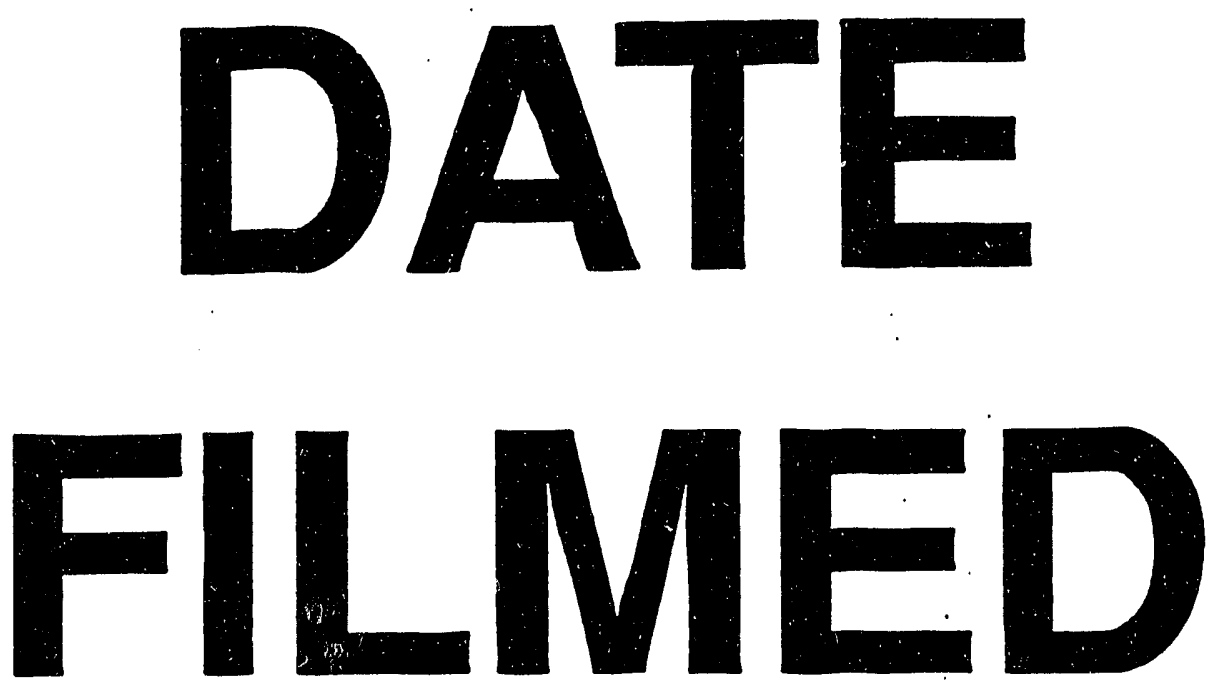

$10 / / 4 / 93$
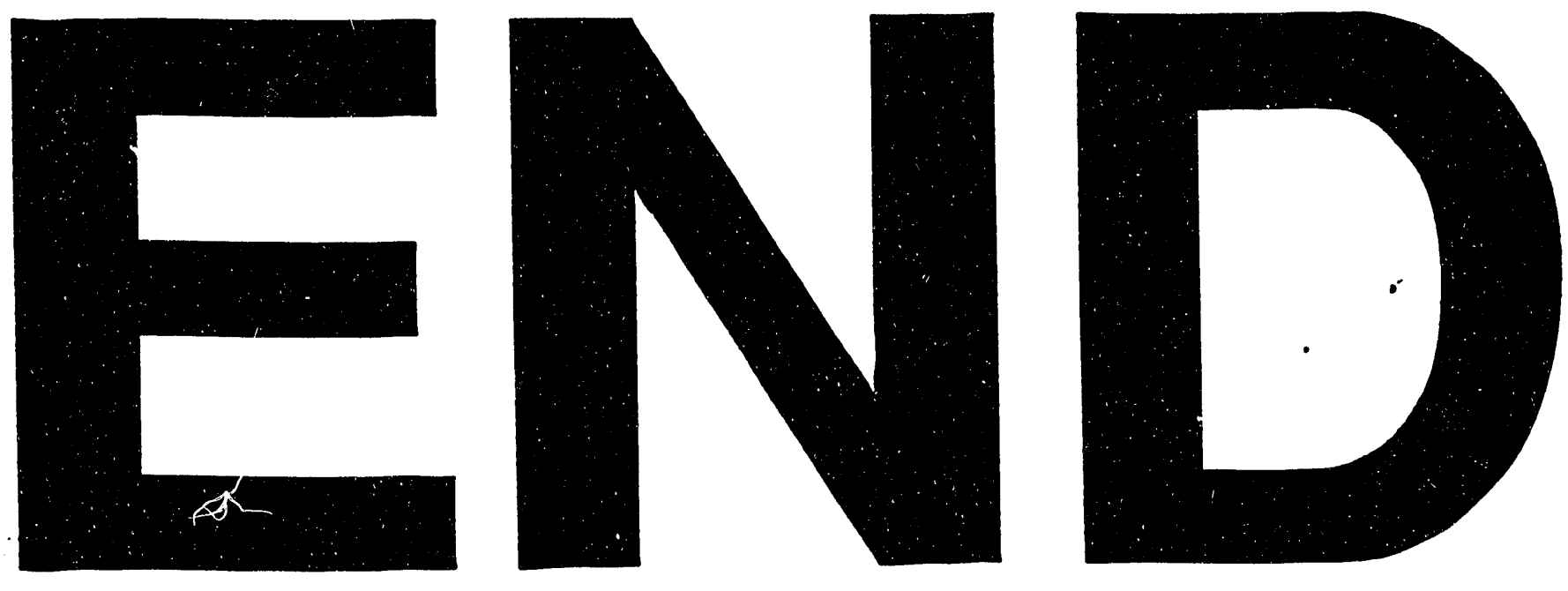
